\title{
Apical mural thrombus: technical pitfalls
}

\author{
K Carpenter, D Adams
}

Since the late 1970s cross sectional echocardiography has been the method of choice for the detection of left ventricular thrombus. This is largely due to the high sensitivity (92-95\%) and specificity (86-88\%) of echocardiography in the detection of left ventricular thrombus. Cross sectional echocardiography also provides an indication of the size, shape, mobility, location, and point of attachment of a thrombus. Most commonly a thrombus will occur in three clinical settings: recent myocardial infarctions, dilated (congestive) cardiomyopathies, and within chronic left ventricular aneurysms. The echocardiographic appearance of a thrombus can vary from a small to large, immobile, mural thrombus (fig 1) to a protruding mobile thrombus, of various sizes, or in some instances multiple thrombi. The most difficult to diagnose is a small, immobile, apical mural thrombus. A small mural thrombus is often less echo dense, and therefore more difficult to distinguish from underlying myocardium.

The diagnosis of left ventricular thrombus is usually based on an underlying wall motion abnormality, and a cardiac mass that can be distinguished from the surrounding myocardium through different acoustic characteristics. For the purpose of this paper our definition of an apical mural thrombus is a distinct mass of echoes, most commonly seen in the apex throughout the cardiac cycle, and in more than one view.

Mural thrombi are most commonly seen between six and 10 days following an acute myocardial infarction (MI). They occur at the left ventricular apex and are more common following an anterior wall infarction, since anterior MIs involve more of the apex (fig 2). This thrombus usually develops on a dyskinetic
Laboratory, Duke

University Medical Center, Durham, NC 27710, USA

K Carpenter

D Adams

Correspondence to: Dr Carpenter.
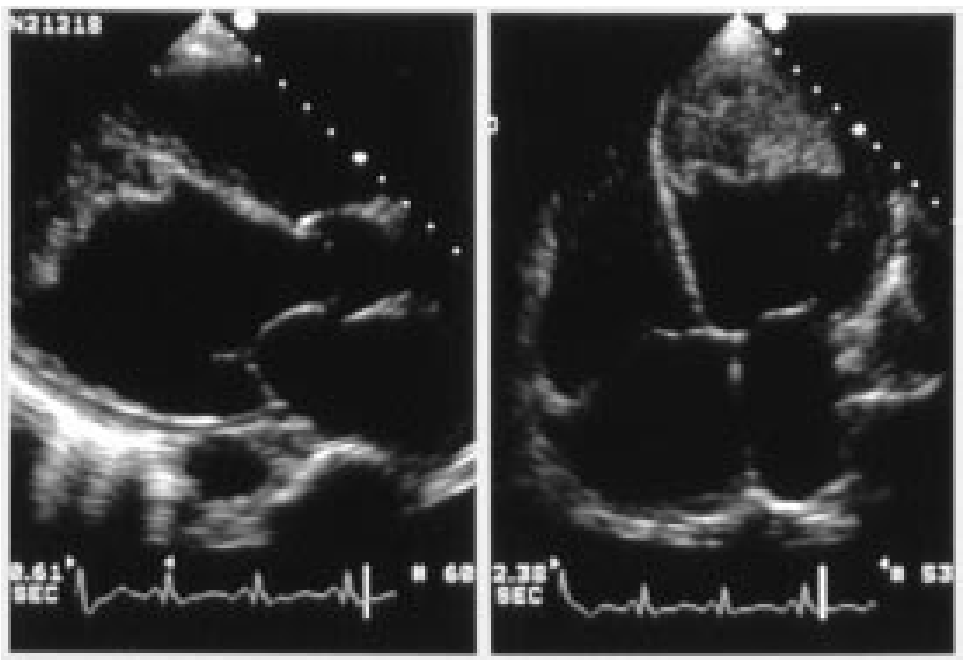

Figure 1 A very large, immobile, apical thrombus is seen in a patient with an ischaemic cardiomyopathy. This thrombus is seen in both a parasternal long axis view (left) and an apical four chamber view (right). or akinetic area or within a left ventricular aneurysm (fig 3). In the absence of a regional wall motion abnormality the diagnosis of left ventricular thrombus should be made with care. A mural thrombus can also occur with large inferior infarctions, especially if an aneurysm develops.

With improved echocardiographic instrumentation and higher frequency transducers, static blood is often seen in patients with low cardiac output. This spontaneous echo contrast (smoke) is thought to result from the agglutination of red blood cells and is most often seen in patients with dilated, hypocontractile ventricles (fig 4). "Smoke" may be seen in conjunction with left ventricular thrombi, and when present indicates that a careful examination of the left ventricle is required. In patients with mitral stenosis, especially with transoesophageal echocardiography, spontaneous echo contrast will often be seen in the left atrium and may indicate the presence of a left atrial thrombus.

Thrombus formation in the right ventricle is seen infrequently and is usually associated with a right ventricular infarction. There have also been reported cases of right ventricular thrombi caused by blunt trauma such as with deceleration injuries in motor vehicle accidents. Again, these types of right ventricular thrombus usually form on a myocardial wall that is moving abnormally.

\section{Scanning techniques}

As most left ventricular thrombi occur at the cardiac apex, the apical and subxiphiod windows are the most useful. It can also be helpful to obtain multiple views, often unconventional or foreshortened, with the transducer angled slightly to visualise the apex better. As high a frequency probe as possible should be used. A $5 \mathrm{MHz}$, short focus probe or even a $7 \mathrm{MHz}$ is ideal. The higher frequency helps in giving better resolution for differentiating between a thrombus and the underlying myocardium. Often an apical thrombus can be obscured by noise artefact; by using a higher frequency transducer there will be less noise artefact introduced in most patients. Noise artefact can also be reduced by decreasing the focal zone to the depth of interest which can improve lateral resolution. Of course, with a higher frequency probe there is less penetration, but that is not an issue since you are only looking at the left ventricular apex at a shallow depth $(4-6 \mathrm{~cm})$. Always use the lowest transmit power and overall gain settings in order to maximise resolution. Low gain settings will also decrease the occurrence of reverb or ring down artefacts, as will sometimes changing scan depth or angle. Finally, document the 

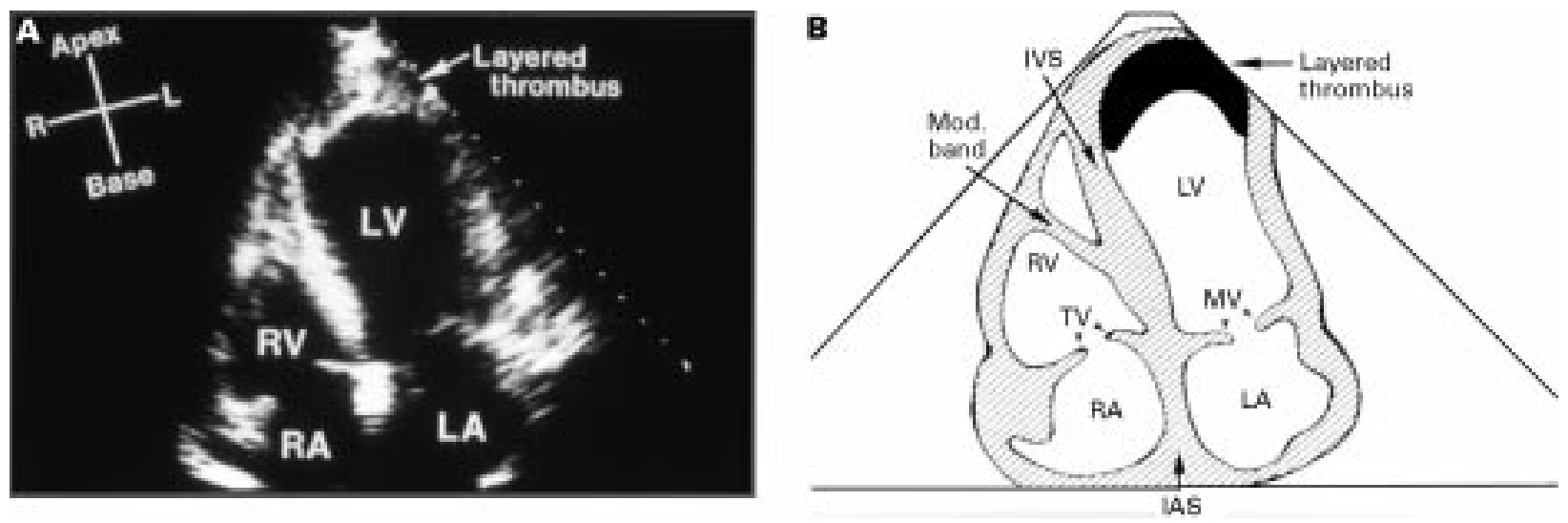

Figure 2 (A) Apical four chamber view showing a layered, mural thrombus causing the apex to appear foreshortened. (B) A schematic drawing identifies more detailed anatomy.

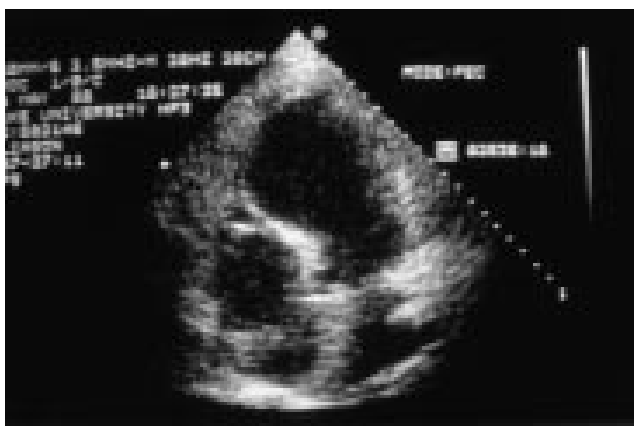

Figure 3 Apical four chamber view in a patient with a left ventricular aneurysm. The distal septum and apical wall appears thin and bulging during systole. This is a typical appearance of a true aneurysm of the left ventricle.

presence of any mass from more than one echocardiographic view and use non-standard views in order to explore the entire left ventricular apex or area in question.

\section{Limitations}

Many of these mural thrombi are small or layered, and difficult to differentiate from underlying myocardium. A new thrombus may not be very echogenic and could be missed without a thorough echocardiographic examination. Another problem is in patients with poor sound transmission in which the left ventricular apex is just not seen. Large left sided trabeculae, anomalous bands, papillary muscles, and reverberations from strong, near field apical echoes which ring down into the left ventricular cavity further confuse the diagnosis of a
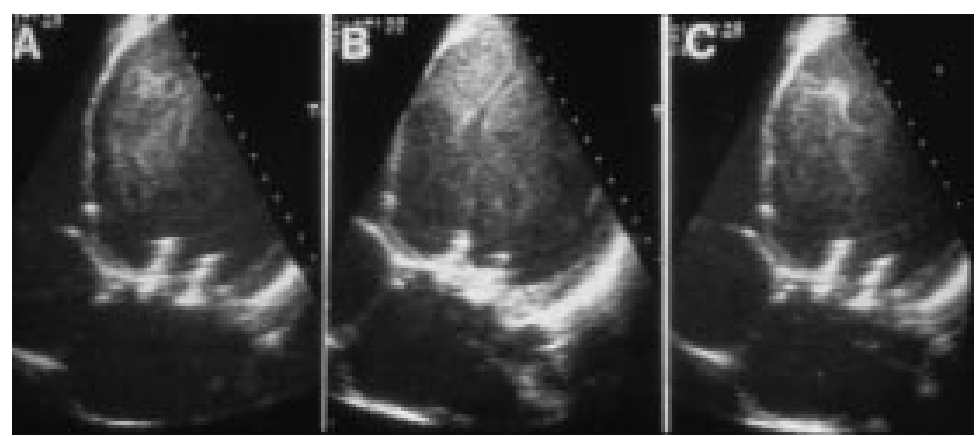

Figure 4 Apical four chamber view in a patient with a bioprosthetic mitral valve and spontaneous echo contrast seen in the left ventricle. The swirling of red blood cells in the sequence $A-C$ is often seen in low cardiac output states and requires careful investigation for an underlying mural thrombus. mural thrombus. One further limitation with echocardiographic evaluation of an apical thrombus is there is no absolute way to tell if a mass seen in the apex is a thrombus or another type of cardiac mass. Echocardiography can show the location, size, shape, mobility, and point of attachment of masses, but not histological evaluation. Ultrasonic tissue characterisation of cardiac masses is a new area of interest but it is not used clinically at this time.

\section{Suggestions for overcoming limitations}

One method is to evaluate the thickness of the myocardium in the areas surrounding the apex. Often walls are thinned and akinetic, and a thrombus will make the apex appear unusually thick.

When evaluating patients with poor image quality, harmonic imaging can be very helpful with visualisation of the apex. Echogenic contrast enhancement agents can also delineate endocardial borders in patients with suboptimal images.

\section{Differential diagnosis}

ANOMALOUS BANDS

Anomalous bands, sometimes called false chordae/tendons, are usually seen in the left ventricular apical area. They commonly extend between the papillary muscles or between a papillary muscle and the left ventricular wall. With cross sectional echocardiography these false tendons appear as a linear echo dense structure, which might be confused with left ventricular thrombi. At times a thicker, more muscular left ventricular band may also be seen in this same region.

\section{EUSTACHIAN VALVE}

One of the most common variants is the eustachian valve, which is sometimes referred to as the valve of the inferior vena cava (IVC). This valve is located in the right atrium, with attachment to the IVC, and is a remnant of the fetal circulation. In the fetus, the eustachian valve directs the highly oxygenated blood flow from the IVC directly across the foramen ovale to the left atrium, bypassing the fetal lungs. The eustachian valve should regress as the fetus grows, but sometimes in adults the valve is prominent. Clinically it is not thought to be very significant. 
Table 1 Mural thrombus

Occur from 6-10 days postmyocardial infarction Occur most commonly with anterior myocardial infarctions Seen most often in the left ventricular apex

May be layered and difficult to see

Usually have underlying wall motion abnormalities

Echocardiographically, the eustachian valve appears to be a thin, linear, filamentous structure. When prominent, the valve usually moves with the blood flow, and with severe tricuspid regurgitation can prolapse into the inferior vena cava.

CHIARI NETWORK

Another very similar variant is a Chiari network, which is found in $2-3 \%$ of normal individuals. ${ }^{1}$ The Chiari network is also a remnant of the eustachian valve, but as the valve regresses, it forms a web of tissue. The Chiari is located in the same area as the eustachian valve, and is also thin and mobile, but it differs from the eustachian valve in its size. The Chiari is more web like and a larger mass of tissue. The Chiari network is usually attached to the region of the crista terminalis (a prominent muscular ridge located between the IVC and superior vena caval orifices). While the Chiari network has no functional significance there is some danger of catheters (pacemaker, Swan Ganz) becoming entrapped within the network. Chiari himself described thrombus formation within this network.
Table 2 Technique

Look for underlying wall motion abnormalities

Use high frequency, short focused transducers

Decrease depth of field

Use low transmit power and gain

Document mass from more than one view

$2^{\text {nd }}$ harmonics

Adjust focus

Contrast enhancement

MODERATOR BAND

The moderator band located in the lower third of the right ventricle can also be a source of confusion. The moderator band is a ridge of tissue which extends from the intraventricular septum to the base of the anterior papillary muscle. The moderator band carries part of the conducting system of the heart (right branch of the atrioventricular bundle). The moderator band is also a landmark used to help in differentiation of the two ventricles. As the right ventricle dilates or becomes hypertrophied, the moderator band can become more pronounced and may be confused with a right ventricular apical thrombus.

\section{Summary}

A left ventricular thrombus can occur in a variety of clinical settings and, when small, may be difficult to document. Understanding the clinical scenarios of when these thrombi form and how to optimise echocardiographic scanning techniques will increase the sensitivity and specificity of the examination (tables 1 and 2).

1 Goldschlager A, Goldschlager N, Brewster H, et al. Catheter entrapment in a Chiari network involving an atrial septal defect. Chest 1972;62:345. 